\title{
Patients with chronic obstructive pulmonary disease and chronically colonized with Haemophilus influenzae during stable disease phase have increased airway inflammation
}

\author{
This article was published in the following Dove Press journal: \\ International Journal of COPD \\ 4 May 2015 \\ Number of times this article has been viewed
}

\section{Ellen Tufvesson \\ Leif Bjermer \\ Marie Ekberg}

Respiratory Medicine and Allergology, Department of Clinical Sciences, Lund University, Lund, Sweden
Correspondence: Ellen Tufvesson Respiratory Medicine and Allergology, Department of Clinical Sciences, Lund University, Lund 22184, Sweden Tel +464 62227828

Email ellen.tufvesson@med.lu.se
Background: Some patients with chronic obstructive pulmonary disease (COPD) show increased airway inflammation and bacterial colonization during stable phase. The aim of this study was to follow COPD patients and investigate chronic colonization with pathogenic bacteria during stable disease phase, and relate these findings to clinical parameters, inflammatory pattern, lung function, and exacerbations.

Methods: Forty-three patients with COPD were included while in a stable state and followed up monthly until exacerbation or for a maximum of 6 months. The patients completed the Clinical COPD Questionnaire and Medical Research Council dyspnea scale questionnaires, and exhaled breath condensate was collected, followed by spirometry, impulse oscillometry, and sputum induction.

Results: Ten patients were chronically colonized (ie, colonized at all visits) with Haemophilus influenzae during stable phase. These patients had higher sputum levels of leukotriene $\mathrm{B}_{4}$ $(P<0.001), 8$-isoprostane $(P=0.002)$, myeloperoxidase activity $(P=0.028)$, and interleukin- 8 $(P=0.02)$ during stable phase when compared with other patients. In addition, they had lower forced vital capacity $(P=0.035)$ and reactance at $5 \mathrm{~Hz}(P=0.034)$, but there was no difference in forced expiratory volume in 1 second $\left(\mathrm{FEV}_{1}\right), \mathrm{FEV}_{1} \%$ predicted, forced vital capacity $\%$ predicted, exhaled breath condensate biomarkers, C-reactive protein, or Clinical COPD Questionnaire and Medical Research Council dyspnea scale results. Three patients had intermittent colonization (colonized at only some visits) of $H$. influenzae during stable phase, and had lower levels of inflammatory biomarkers in sputum when compared with the chronically colonized patients. The difference in airway inflammation seen during stable phase in patients chronically colonized with $H$. influenzae was not observed during exacerbations.

Conclusion: Some COPD patients who were chronically colonized with $H$. influenzae during stable phase showed increased airway inflammation and reduced lung volumes when compared with non-chronically colonized patients.

Keywords: chronic obstructive pulmonary disease, inflammation, biomarker, colonization, sputum, spirometry

\section{Introduction}

Chronic obstructive pulmonary disease (COPD) is characterized by an accelerated decline in lung function, progressive airway inflammation, and exacerbations. Often COPD patients also have increased airway inflammation during stable phase. The degree of airway inflammation is positively related to the severity of airway obstruction, with more bronchial inflammation in patients who have lower forced expiratory 
volume in 1 second $\left(\mathrm{FEV}_{1}\right) .{ }^{1}$ Further, higher levels of airway inflammation have been associated with a greater rate of decline in $\mathrm{FEV}_{1}{ }^{2}{ }^{2}$

Bacterial colonization in COPD is an important factor in progression of the disease, and both bacterial and viral infections are common comorbidities during COPD exacerbations. ${ }^{3}$ Bacteria have also been isolated from the lower airways of patients with stable COPD, and FEV decline has $_{1}$ been related to increased bacterial load in the airways. Even in the absence of clinical exacerbation, colonization by bacterial pathogens in stable COPD is associated with a clinically significant moderate increase in daily symptoms. ${ }^{4}$ Increasing bacterial load in the airways of patients with stable COPD has been shown to be associated with an accelerated decline in $\mathrm{FEV}_{1}$ and greater airway inflammation, which is often characterized by a neutrophilic response, such as increased levels of interleukin (IL)-8 and leukotriene $\mathrm{B}_{4}\left(\mathrm{LTB}_{4}\right){ }^{5-7}$ Specifically, markers of inflammation have been shown to increase progressively with increasing bacterial load. ${ }^{8}$

Haemophilus influenzae has been observed to be the most prevalent type of bacterium in patients with stable COPD. ${ }^{6,7}$ Sputum levels of myeloperoxidase and IL-8 are higher in COPD patients colonized with $H$. influenzae as compared with non-colonized patients, suggesting a more marked inflammatory response due to $H$. influenzae colonization. ${ }^{9,10}$

Some COPD patients have persistent bacterial colonization in the airways (referred to as chronic colonization) between exacerbations. This chronic colonization may contribute to the morbidity of COPD through persistent airway inflammation, even in the stable clinical state. Currently, there is no long-term prospective study involving repeated measurements of airway inflammation, lung function, and bacterial colonization in such patients. Therefore, we performed sputum bacteriology in patients with COPD at their monthly visits and examined its relationship to airway inflammation. Inflammatory biomarker levels in sputum from these patients in stable phase until exacerbation has been described previously. ${ }^{11}$ The aim of this study was to follow COPD patients at several visits and investigate how chronic colonization with pathogenic bacteria during stable disease phase relates to clinical parameters, inflammatory pattern, lung function, and exacerbations.

\section{Materials and methods Subjects}

Forty-three patients with COPD and at least one exacerbation requiring antibiotics during the previous year were included.
All patients were attending our specialist outpatient COPD clinic at Lund University Hospital, and were recruited for the study by a pulmonologist. Study participants had no history of lung cancer or asthma. The subjects were free from any respiratory infection for 6 weeks prior to the study and had not been treated for an exacerbation in the 3 weeks prior to the study. Three patients were excluded due to initiation of immunosuppressive medication (such as methotrexate and azathioprine) during the study.

\section{Study design}

The study participants were included in their stable state, and were followed up monthly for up to 6 months. The patients came for an extra visit (prior to the scheduled visit) when exacerbating. An exacerbation was defined as an acute change in the patient's baseline dyspnea, cough, and/ or sputum beyond day-to-day variability sufficient to warrant a change in therapy (according to American Thoracic Society/European Respiratory Society guidelines, 2004). The study was approved by the Regional Ethical Review Board in Lund, and all participants signed their written informed consent. A physical examination was performed before the study started. At all visits, patients inhaled $5 \mathrm{mg}$ of salbutamol (Ventolin $^{\mathrm{TM}}$, GlaxoSmithKline, London UK) and $0.5 \mathrm{mg}$ ipratropium (Atrovent ${ }^{\mathrm{TM}}$, Boehringer Ingelheim, Ingelheim, Germany) prior to investigations. The patients completed two questionnaires, ie, the Clinical COPD Questionnaire (CCQ) ${ }^{12}$ and the Medical Research Council (MRC) dyspnea scale. Thereafter, exhaled breath condensate (EBC), spirometry, impulse oscillometry (IOS), and sputum induction were performed. Each parameter value of stable phase was calculated as an average value of all visits during stable phase.

\section{Spirometry with IOS}

All subjects performed spirometry using the Jaeger MasterScreen (Erich Jaeger GmbH, Würzburg, Germany). The reference values established by Crapo et al were used. ${ }^{13}$ Spirometry was followed by IOS using the Jaeger MasterScreen impulse oscillometry system. The subjects used nose clips and were told to press the palms of their hands against their cheeks to decrease the upper airway's shunt. For 30 seconds, oscillometric pressure impulses were superimposed on tidal breathing, having a pulse sequence of 5 per second and a frequency spectrum of 5-35 Hz. Airway resistance at $5 \mathrm{~Hz}$ and $20 \mathrm{~Hz}$ (R5 and R20, respectively), reactance at $5 \mathrm{~Hz}$, resonant frequency, and area of reactance integrated from $5 \mathrm{~Hz}$ to resonant frequency (AX) were determined, and R5-R20 was calculated. 


\section{EBC collection and analysis}

EBC was collected using ECoScreen (Jaeger), and to eliminate loss of biomarkers due to absorbance, all plastic surfaces were coated with $1 \%$ bovine serum albumin and $0.01 \%$ Tween 20 , as previously described. ${ }^{14}$ Subjects rinsed their mouths with water and breathed tidally for 15 minutes while wearing a nose clip. The condensates were stored at $-70^{\circ} \mathrm{C}$ until analysis.

The activity of $\alpha$-amylase was analyzed using the EnzChek Ultra amylase assay kit (E33651) from Molecular Probes (Eugene, OR, USA) to exclude contamination with saliva. ${ }^{15}$ Due to low concentrations in $\mathrm{EBC}$, samples were concentrated (8-10 times) by freeze-drying and resolved in the respective assay buffer. The final concentrations were calculated from specific freeze-dried volumes. $\mathrm{LTB}_{4}$ and 8-isoprostane were analyzed using an enzyme-linked immunosorbent assay kit from Cayman Chemicals (Ann Arbor, MI, USA).

\section{Sputum induction, processing, and analysis}

Sputum was induced by inhalation of nebulized $0.9 \% \mathrm{NaCl}$ for $0.5,1,2$, and 4 minutes, followed by $4.5 \% \mathrm{NaCl}$ for 0.5 , 1,2 , and 4 minutes. Sputum induction was continued until an adequate sample volume was obtained. ${ }^{16}$ The sputum sample was divided into two aliquots.

Sputum purulence was determined using a colorimetric scale. ${ }^{17}$ One aliquot of the sputum was sent for routine bacterial culture at the Clinical Microbiology Department, Lund University Hospital. Colonization was defined as a positive culture for any airway pathogen. In the other aliquot of sputum, sputum plugs were picked out, treated with four volumes of $0.65 \mathrm{mM}$ dithiothreitol in phosphate-buffered saline for 1 hour at $4^{\circ} \mathrm{C}$, followed by addition of four volumes of phosphate-buffered saline. The sputum was filtered $(60 \mu \mathrm{m})$ and centrifuged (1,000 $\mathrm{g}$ for 5 minutes), and the supernatant was frozen for later analysis.

Sputum was analyzed for $\mathrm{LTB}_{4}$ and 8-isoprostane (similar to EBC). Before analysis of subsequent protein assays, the sputum was dialyzed in phosphate-buffered saline to eliminate the remaining dithiothreitol. IL-8 was measured using a Quantikine assay (R\&D Systems, Minneapolis, MN, USA). Myeloperoxidase activity was measured according to Axelsson et al ${ }^{18}$ by adding 3,3',5,5'-tetramethylbenzidine and hydrogen peroxide to the sputum, stopping the reaction with $2 \mathrm{M} \mathrm{H}_{2} \mathrm{SO}_{4}$ after 3 minutes, and analysis at $450 \mathrm{~nm}$. Horseradish peroxidase was used as the standard.

All values were adjusted to the total amount of protein in sputum, measured using the Bio-Rad protein assay (Bio-Rad
Laboratories Inc, Hercules, CA, USA) and presented as amount per microgram of total protein to remove differences due to heterogeneity of the sputum.

\section{Statistical analysis}

The Mann-Whitney $U$-test for unpaired samples was used to compare groups of patients (chronically colonized versus non-chronically colonized). Statistical comparisons between groups (chronically colonized versus intermittently colonized versus no growth) were performed using the Kruskal-Wallis test for unpaired samples. The chi-squared test was used for group comparisons (numbers of exacerbations in chronically colonized versus not chronically colonized patients). All statistical calculations were performed using Statistical Package for the Social Sciences version 20.0 (SPSS Inc, Chicago, IL, USA). The mean value for each parameter at stable phase was calculated for each individual patient. The data are thereafter shown as the median value (interquartile range) of the individual mean values. The level of statistical significance was set at $P \leq 0.05$.

\section{Results}

\section{Patients}

A total of 201 patient visits were included in this study, with a median (interquartile range) of five (3-7) visits per patient (either until exacerbation or until 6 months). No adequate sputum could be obtained from nine patients during stable phase, but five could expectorate sputum during an exacerbation. Twelve patients did not have any positive pathogenic bacterial cultures (H. influenzae, Pseudomonas aeruginosa, Staphylococcus aureus, Streptococcus pneumonia, and Moraxella catarrhalis) during the study period.

\section{Chronic colonization}

Thirteen patients were colonized with the same type of bacteria at all visits during stable phase, and were defined as being chronically colonized. Ten patients were chronically colonized by $H$. influenzae, two by $S$. aureus, and one by $P$. aeruginosa. No patient was chronically colonized by $S$. pneumoniae or $M$. catarrhalis. The ten patients who were chronically colonized by $H$. influenzae made a median (interquartile range) of four (3-7) visits.

\section{Chronic colonization with $H$. influenzae}

Patients chronically colonized with $H$. influenzae during stable phase (Table 1) had significantly higher levels of inflammatory biomarkers in sputum compared with patients not chronically colonized with $H$. influenzae (Figure 1 ); 
Table I Patient characteristics

\begin{tabular}{lll}
\hline & $\begin{array}{l}\text { Chronically colonized } \\
\text { with H. influenzae } \\
\mathbf{n = 1 0}\end{array}$ & $\begin{array}{l}\text { Not chronically } \\
\text { colonized with } \\
\text { H. influenzae } \mathbf{n = 2 ~ I ~}\end{array}$ \\
\hline Females/males, n & $6 / 4$ & $9 / 12$ \\
Age, years & $68(63-70)$ & $65(57-73)$ \\
Smokers/ex-smokers, n & $2 / 8$ & $6 / 15$ \\
Pack-years & $39(32-44)$ & $40(35-50)$ \\
Exacerbates, n & 7 & 11 \\
GOLD I, n & 0 & 1 \\
GOLD 2, n & 2 & 6 \\
GOLD 3, n & 6 & 9 \\
GOLD 4, n & 2 & 5 \\
\hline
\end{tabular}

Notes: All patients had FEV/FVC $<0,7$, and GOLD stages were defined as GOLD I (FEV $1 \geq 80 \%$ of predicted normal), GOLD $2\left(50 \geq \mathrm{FEV}_{1}<80 \% \mathrm{p}\right)$, GOLD $3(30 \geq \mathrm{FEV},<50 \% \mathrm{p})$ and GOLD $4\left(\mathrm{FEV}_{1}<30 \% \mathrm{p}\right)$. Exacerbates = number of patients that exacerbate during the study.

Abbreviations: $\mathrm{FEV}_{1}$, forced expiratory volume in I second; GOLD, Global Initiative for Chronic Obstructive Lung Disease; $H$. influenzae, Haemophilus influenzae; \%p, \% of predicted normal.

$\mathrm{LTB}_{4}(P<0.001), 8$-isoprostane $(P=0.002)$, myeloperoxidase activity $(P=0.028)$, and IL-8 $(P=0.02)$. In addition, these patients had lower forced vital capacity (FVC) $(P=0.035)$ and a tendency for lower $\mathrm{FEV}_{1}(P=0.060)$, but no difference in $\mathrm{FEV}_{1} \%$ predicted or $\mathrm{FVC} \%$ predicted (Figure $2 \mathrm{~A}$ ). IOS parameters reflecting primarily the peripheral airways, such as reactance at $5 \mathrm{~Hz}(P=0.034)$, were also higher in patients chronically colonized with $H$. influenzae (Figure 2B). In addition, there was a tendency for increased resistance and reactance in other peripheral IOS parameters, such as R5, R5-R20, AX, and resonant frequency, although these results were not significant. No difference was observed in inflammatory biomarkers measured in EBC (Figure 3A) or in clinical parameters, ie, C-reactive protein (CRP), CCQ, or MRC scores (Figure 3B). There were no differences in age, sex, smoking status, or oxygen saturation. There was a tendency, albeit not statistically significant, for increased frequency of exacerbation during the study among patients chronically colonized with $H$. influenzae; seven of ten (70\%) chronically colonized patients exacerbated compared with eleven of 21 (52\%) non-chronically colonized patients (Figure 4).

\section{Intermittent colonization with $H$. influenzae}

In addition to the 13 patients who were chronically colonized at all visits, six patients were colonized at only some (recurring) visits (defined as intermittent colonization). Three of these patients had intermittent colonization with H. influenzae. Of note, these three patients with intermittent H. influenzae colonization showed significantly lower levels of $\operatorname{LTB}_{4}(P=0.043)$ and IL-8 $(P=0.043)$, and a tendency for lower 8 -isoprostane $(P=0.176)$ and myeloperoxidase activity $(P=0.128)$, compared with patients chronically colonized with $H$. influenzae (Figure 5A-D). In addition, patients with intermittent $H$. influenzae colonization had lower levels of

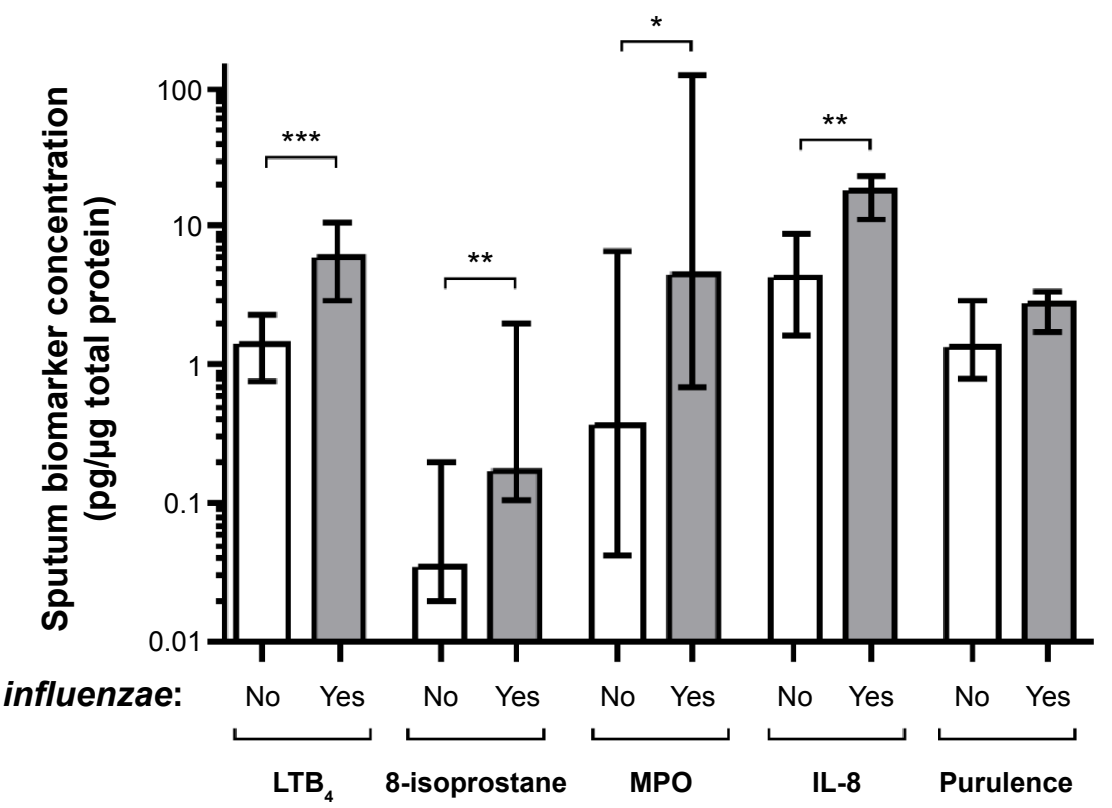

Figure I Concentrations of sputum biomarkers ( $\mathrm{LTB}_{4}$, 8-isoprostane, MPO activity, IL-8) and purulence scores for patients chronically colonized with Haemophilus influenzae (yes) and in patients not chronically colonized with $H$. influenzae (no).

Notes: The graphs show levels of these parameters at stable phase (using the mean of each parameter at all stable visits). The data are presented as the median (interquartile range) and $* P \leq 0.05, * * P \leq 0.01$, $* * * P \leq 0.00 \mathrm{I}$ for comparisons between colonized and non-colonized patients.

Abbreviations: $H$. influenzae, Haemophilus influenzae; $\mathrm{LTB}_{4}$, leukotriene $\mathrm{B}_{4}$; MPO, myeloperoxidase; IL, interleukin. 


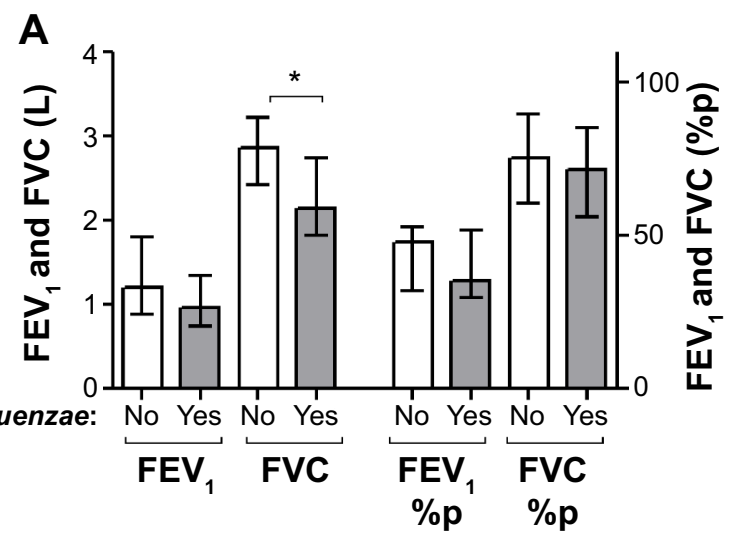

B

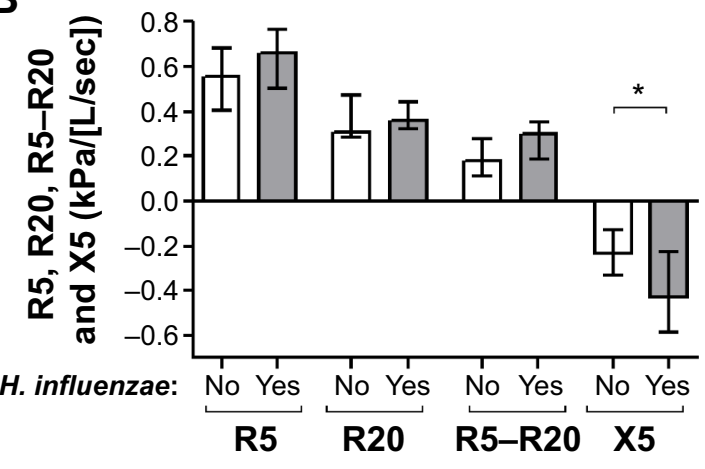

Figure 2 Lung function parameters in patients chronically colonized with $H$. influenzae (yes) and in patients not chronically colonized with $H$. influenzae (no) as measured by (A) spirometry and (B) impulse oscillometry.

Notes: The graphs show levels of these parameters at stable phase (using the mean of each parameter at all stable visits). The data are presented as the median (interquartile range) and $* P \leq 0.05$ for comparison between colonized and non-colonized patients.

Abbreviations: $H$. influenzae, Haemophilus influenzae; \%p, percent predicted; FEV $_{1}$, forced expiratory volume in I second; FVC, forced vital capacity.

8-isoprostane in $\operatorname{EBC}(P=0.018)$. However, no difference was observed for sputum purulence (Figure 5E), spirometry, IOS, or clinical parameters.

When comparing patients with intermittent colonization of $H$. influenzae and patients not colonized with any pathogenic bacteria during the study ( $\mathrm{n}=12$ ), no differences could be seen in sputum or EBC biomarkers, spirometry, IOS, or clinical parameters.

\section{Colonization at exacerbation}

Twenty-five patients (63\%) exacerbated within the 6-month study period at visit $3(2-5)$. Five patients did not manage to come for the exacerbation visit or could not expectorate any sputum. Of the remaining 20 patients, 16 were colonized with pathogenic bacteria at exacerbation; 12 had H. influenzae colonization (four were only colonized at exacerbation), three had $S$. aureus (one patient was only colonized at exacerbation), two had
P. aeruginosa (one patient was only colonized at exacerbation), six had S. pneumoniae (five patients were only colonized at exacerbation), and five had $M$. catarrhalis (all were only colonized at exacerbation) during exacerbation. Another interesting finding was co-colonization of $S$. aureus and $S$. pneumoniae during exacerbation, with all patients colonized with $S$. aureus being also colonized with $S$. pneumoniae.

In contrast with the overall higher level of airway inflammation in sputum biomarkers seen during stable phase in patients chronically colonized with $H$. influenzae, these patients did not have higher levels of airway inflammation during exacerbation (except for 8 -isoprostane, $P=0.021$ ) compared with patients not chronically colonized with H. influenzae.

At exacerbation also some of the non-chronically colonized patients were colonized with $H$. influenzae. All these patients that were colonized with $H$. influenzae during
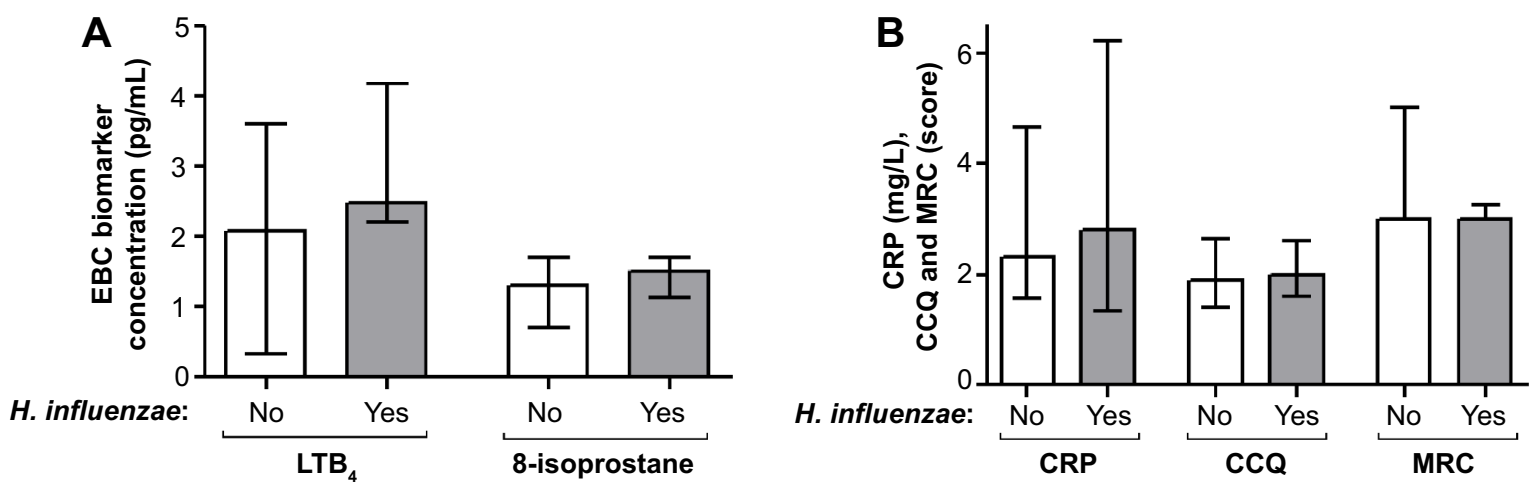

Figure 3 Concentrations of EBC biomarkers (A) and clinical parameters (B) in patients chronically colonized with $H$. influenzae (yes) and in patients not chronically colonized with $H$. influenzae (no).

Notes: The graphs show levels of these parameters at stable phase (using the mean of each parameter at all stable visits). The data are presented as the median (interquartile range).

Abbreviations: $H$. influenzae, Haemophilus influenzae; $\mathrm{LTB}_{4}$, leukotriene $\mathrm{B}_{4}$; MRC, Medical Research Council dyspnea scale; CRP, C-reactive protein; CCQ, Clinical COPD Questionnaire; EBC, exhaled breath condensate. 


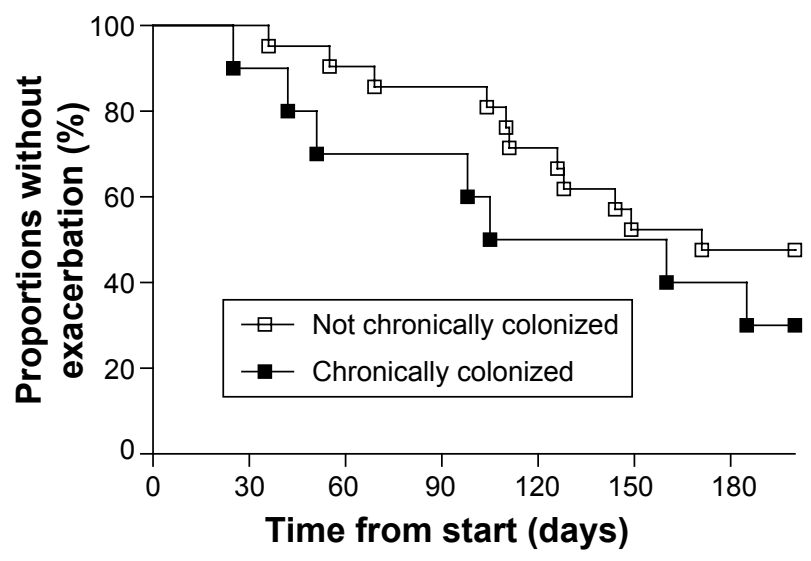

Figure 4 Kaplan-Meier curve of the proportion of patients who exacerbated during the 6-month study period.

exacerbation $(\mathrm{n}=12)$ showed significantly higher levels of IL-8 $(P=0.017)$ in sputum and 8-isoprostane in EBC $(P=0.041)$ as compared with patients not colonized with $H$. influenzae during exacerbation. No significant changes in other sputum or EBC biomarkers, spirometry, IOS, or clinical parameters were found between the patient groups.

\section{Discussion}

In this study, COPD patients were followed over several visits during stable phase, and bacterial colonization pattern was observed. Some patients were colonized with the same pathogenic bacteria at all visits, so were defined as chronically colonized. COPD patients chronically colonized with H. influenzae had increased airway inflammation, measured by sputum biomarkers, during stable phase as compared with non-chronically colonized COPD patients. Biomarkers for neutrophil activity (eg, $\mathrm{LTB}_{4}$, myeloperoxidase activity, and IL-8) and oxidative stress (eg, 8-isoprostane) were increased in such patients. These chronically colonized patients also had lower FVC and reactance at $5 \mathrm{~Hz}$, but otherwise there were no differences in spirometry or IOS parameters, in EBC inflammatory biomarkers, or in clinical parameters (including CRP, CCQ, and MRC scores). Interestingly, there was a difference between patients with chronic and intermittent colonization (patients colonized at only some visits) because those with only intermittent $H$. influenzae colonization showed significantly lower levels of sputum inflammatory biomarkers, and did not differ from those with no colonization at all.

This was a prospective study with repeated visits over 6 months or until exacerbation. Repeated measurements of airway inflammation and bacterial colonization in COPD patients during stable disease phase have not been reported previously. We believe that following the patients for up to 6 months has the advantage of obtaining an overall picture of chronic colonization as opposed to other studies that have presented only one-time snapshots comparing intermittently colonized patients with patients that are not colonized at the moment. ${ }^{5,8-10}$

Patients with chronic $H$. influenzae colonization had higher levels of inflammatory biomarkers in sputum during stable phase compared with patients intermittently colonized with $H$. influenzae. This shows the consequence of a permanent bacterial occurrence, which is apparently related to increased airway inflammation. This could possibly be a question of bacterial load, and those patients not showing culture at all visits in fact had only low levels of bacteria. ${ }^{19}$ The level of airway inflammation in sputum would reflect the relationship between inflammatory biomarkers and bacterial load, which has been reported previously. ${ }^{10}$ One should be aware that traditional bacterial culturing techniques may result in false negatives. An alternative method to identify these bacteria may be through polymerase chain reaction, although this is also not an infallible method. ${ }^{20}$ Thus, it cannot be excluded that sputum from some patients gave a false negative result, ie, being colonized with negative culture, which could have affected the overall results.

Another interesting finding was that the number of patients who were chronically colonized with $H$. influenzae was much higher than the number of patients who were chronically colonized with other pathogenic bacteria, which seldom showed a pattern of chronic colonization. The other bacteria showed a more intermittent pattern, or showed colonization mainly during exacerbation.

This study included an exacerbation-prone population of COPD patients (all patients had at least one exacerbation within the previous year). This could have affected the results with regard to levels of inflammatory biomarkers and bacterial colonization. However, it has been shown that COPD patients with frequent and infrequent exacerbations may be indistinguishable in terms of inflammatory biomarkers in sputum..$^{21}$ In addition, we have previously reported that there was no difference in levels of inflammatory biomarkers during stable phase between patients who exacerbated and those who did not exacerbate within 6 months. ${ }^{11}$ We have also previously demonstrated an increase in inflammatory biomarkers during exacerbation. The present study is a post hoc analysis of patients from this previous publication, and the difference in inflammatory biomarkers observed between patients chronically colonized with $H$. influenzae and those not chronically colonized was no longer apparent during exacerbation. This might be due to an overall increase in inflammatory biomarkers being already present at baseline. 

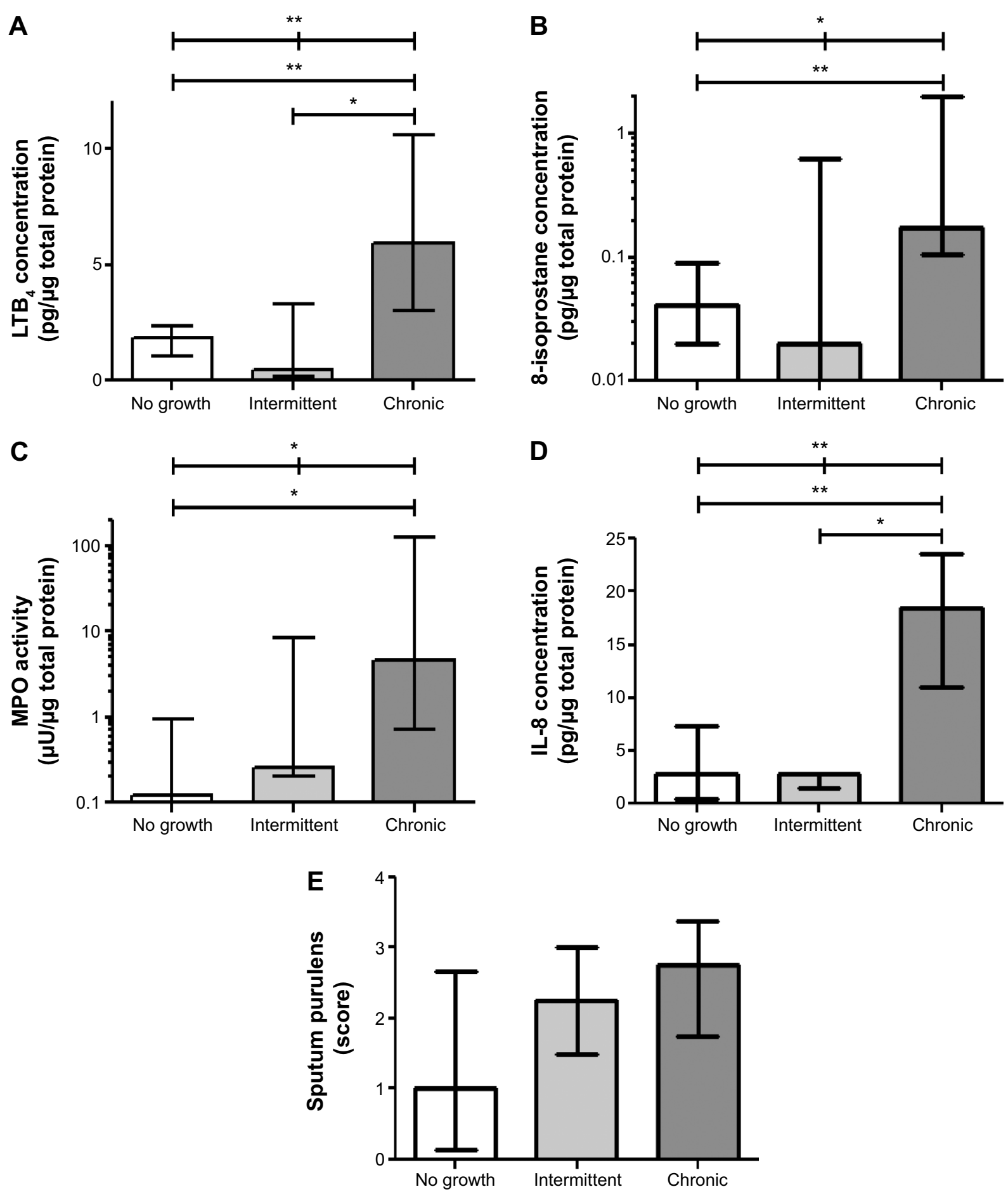

Figure 5 Sputum biomarkers, ie, $\operatorname{LTB}_{4}(\mathbf{A})$, 8-isoprostane (B), MPO activity (C), IL-8 concentration (D), and purulence score (E) in patients chronically colonized with $H$. influenzae (chronic), in patients with intermittent colonization with $H$. influenzae (intermittent) and in patients not colonized by pathogenic bacteria (no growth). The graphs show levels of these parameters at stable phase (using the mean of each parameter at all stable visits). The data are shown as the median (interquartile range) and $* P \leq 0.05$ and $* * P \leq 0.0$ I for comparison between marked patient groups.

Abbreviations: $\mathrm{LTB}_{4}$, leukotriene $\mathrm{B}_{4}$; MPO, myeloperoxidase; IL, interleukin.

The number of patients colonized during stable phase was in accordance with previous investigations of bacterial cultures. ${ }^{22}$ In addition, in terms of bacterial colonization, seven of the ten patients chronically colonized with $H$. influenzae did exacerbate within 6 months. However, the prevalence of viruses during stable phase and exacerbations was not investigated in this study, but could give rise to other inflammatory phenotypes. ${ }^{23}$

This study also shows that patients chronically colonized with $H$. influenzae have poorer lung function as measured by 
FVC and a tendency for lower $\mathrm{FEV}_{1}$. However, there was no difference in $\mathrm{FVC} \%$ predicted or $\mathrm{FEV}_{1} \%$ predicted, which has also been observed previously. ${ }^{8}$ Thus, there appears to be no relationship between $H$. influenzae infection and Global Initiative for Chronic Obstructive Lung Disease (GOLD) stage $1-4 .{ }^{24}$ However, the lower FVC values indicate a relationship with volume-related lung obstruction. There is also a relationship with certain IOS parameters, suggesting an involvement in the peripheral airways. This study was performed before publication of the most recent GOLD classification system (A-D), so we cannot classify our patients according to this system. However, on the basis of MRC scores, we can conclude that all patients were GOLD stage B or D. Further, most patients were included after an exacerbation recovery period of 6 weeks, and because several patients exacerbated during the study, we know that at least 21 patients were in GOLD stage D. Because of lack of data, no conclusions can be drawn from groupings according to the new GOLD classification.

\section{Conclusion}

Some COPD patients were chronically colonized with $H$. influenzae during stable phase, and had increased airway inflammation as measured by sputum biomarkers, compared with COPD patients not chronically colonized with H. influenzae. These chronically colonized patients also had lower FVC, but otherwise there was no difference in EBC inflammatory biomarkers or in clinical parameters, including CRP, CCQ and MRC scores. In contrast, patients with only intermittent $H$. influenzae colonization showed lower levels of airway inflammation, compared with chronically colonized patients, and did not differ compared with patients not colonized with any bacteria.

In chronically colonized patients, there is ongoing silent inflammation that is not detected using clinical routine parameters. It is tempting to conclude that these patients are at higher risk of disease progression, or that COPD patients are more prone to colonization by $H$. influenzae at later stages of the disease. Perhaps monitoring bacterial cultures during stable phase could be advantageous in patients with earlystage COPD to help avoid disease progression.

\section{Acknowledgments}

This work was supported by independent research grants from the Swedish Heart and Lung Foundation, Swedish Research Council, Evy and Gunnar Sandberg's Foundation, and Royal Physiographic Society in Lund. We thank Anna-Karin Juhlin, Harriet Johansson, Tove Alvå, and Oskar
Hermansson at the Lung Clinic, Skåne University Hospital, Lund, for their clinical assistance.

\section{Author contributions}

ET designed the study, performed the analysis, interpreted data, and drafted the manuscript. LB designed the study, interpreted data, and critically revised the manuscript. ME designed the study, included patients, interpreted data, and critically revised the manuscript.

\section{Disclosure}

The authors report no conflicts of interest in this work.

\section{References}

1. Di SA, Capelli A, Lusuardi M, et al. Severity of airflow limitation is associated with severity of airway inflammation in smokers. Am J Respir Crit Care Med. 1998;158(4):1277-1285.

2. Stanescu D, Sanna A, Veriter C, et al. Airways obstruction, chronic expectoration, and rapid decline of FEV1 in smokers are associated with increased levels of sputum neutrophils. Thorax. 1996;51(3):267-271.

3. Decramer M, Janssens W, Miravitlles M. Chronic obstructive pulmonary disease. Lancet. 2012;379(9823):1341-1351.

4. Desai H, Eschberger K, Wrona C, et al. Bacterial colonization increases daily symptoms in patients with chronic obstructive pulmonary disease. Ann Am Thorac Soc. 2014;11(3):303-309.

5. Banerjee D, Khair OA, Honeybourne D. Impact of sputum bacteria on airway inflammation and health status in clinical stable COPD. Eur Respir J. 2004;23(5):685-691.

6. Wilkinson TM, Patel IS, Wilks M, Donaldson GC, Wedzicha JA. Airway bacterial load and FEV1 decline in patients with chronic obstructive pulmonary disease. Am J Respir Crit Care Med. 2003;167(8): 1090-1095.

7. Zhang M, Li Q, Zhang XY, Ding X, Zhu D, Zhou X. Relevance of lower airway bacterial colonization, airway inflammation, and pulmonary function in the stable stage of chronic obstructive pulmonary disease. Eur J Clin Microbiol Infect Dis. 2010;29(12):1487-1493.

8. Hill AT, Campbell EJ, Hill SL, Bayley DL, Stockley RA. Association between airway bacterial load and markers of airway inflammation in patients with stable chronic bronchitis. Am J Med. 2000; 109(4):288-295.

9. Bresser P, Out TA, van Alphen L, Jansen HM, Lutter R. Airway inflammation in nonobstructive and obstructive chronic bronchitis with chronic Haemophilus influenzae airway infection. Comparison with noninfected patients with chronic obstructive pulmonary disease. Am J Respir Crit Care Med. 2000;162(3 Pt 1):947-952.

10. Singh R, MacKay AJ, Patel A, et al. Inflammatory thresholds and the species-specific effects of colonising bacteria in stable chronic obstructive pulmonary disease. Respir Res. 2014;15(1):114.

11. Tufvesson E, Ekberg M, Bjermer L. Inflammatory biomarkers in sputum predict COPD exacerbations. Lung. 2013;191(4):413-416.

12. van der Molen T, Willemse BW, Schokker S, ten Hacken NH, Postma DS, Juniper EF. Development, validity and responsiveness of the Clinical COPD Questionnaire. Health Qual Life Outcomes. 2003;1:13.

13. Crapo RO, Morris AH, Gardner RM. Reference spirometric values using techniques and equipment that meet ATS recommendations. Am Rev Respir Dis. 1981;123(6):659-664.

14. Tufvesson E, Bjermer L. Methodological improvements for measuring eicosanoids and cytokines in exhaled breath condensate. Respir Med. 2006;100(1):34-38.

15. Tufvesson E, van Weele LJ, Ekedahl H, Bjermer L. Levels of cysteinylleukotrienes in exhaled breath condensate are not due to saliva contamination. Clin Respir J. 2010;4(2):83-88. 
16. Tufvesson E, Aronsson D, Bjermer L. Cysteinyl-leukotriene levels in sputum differentiate asthma from rhinitis patients with or without bronchial hyperresponsiveness. Clin Exp Allergy. 2007;37(7):1067-1073.

17. Allegra L, Blasi F, Diano P, et al. Sputum color as a marker of acute bacterial exacerbations of chronic obstructive pulmonary disease. Respir Med. 2005;99(6):742-747.

18. Axelsson JB, Akbarshahi H, Said K, Malmstrom A, Andersson R. Proposed protective mechanism of the pancreas in the rat. $J$ Inflamm (Lond). 2010;7:24.

19. Murphy TF, Brauer AL, Schiffmacher AT, Sethi S. Persistent colonization by Haemophilus influenzae in chronic obstructive pulmonary disease. Am J Respir Crit Care Med. 2004;170(3):266-272.

20. Garcha DS, Thurston SJ, Patel AR, et al. Changes in prevalence and load of airway bacteria using quantitative PCR in stable and exacerbated COPD. Thorax. 2012;67(12):1075-1080.
21. Gompertz S, Bayley DL, Hill SL, Stockley RA. Relationship between airway inflammation and the frequency of exacerbations in patients with smoking related COPD. Thorax. 2001;56(1):36-41.

22. Patel IS, Seemungal TA, Wilks M, Lloyd-Owen SJ, Donaldson GC, Wedzicha JA. Relationship between bacterial colonisation and the frequency, character, and severity of COPD exacerbations. Thorax. 2002;57(9):759-764.

23. Bafadhel M, McKenna S, Terry S, et al. Acute exacerbations of chronic obstructive pulmonary disease: identification of biologic clusters and their biomarkers. Am J Respir Crit Care Med. 2011;184(6):662-671.

24. Rodriguez-Roisin R, Vestbo J, Anzueto A, et al. Global strategy for the Diagnosis, Management and Prevention of Chronic Obstructive Pulmonary Disease, Revised 2011. Global Initiative for Chronic Obstructive Lung Disease. Available from: http://www.goldcopd.org/. Accessed April 9, 2015
International Journal of COPD

\section{Publish your work in this journal}

The International Journal of COPD is an international, peer-reviewed journal of therapeutics and pharmacology focusing on concise rapid reporting of clinical studies and reviews in COPD. Special focus is given to the pathophysiological processes underlying the disease, intervention programs, patient focused education, and self management protocols

\section{Dovepress}

This journal is indexed on PubMed Central, MedLine and CAS. The manuscript management system is completely online and includes a very quick and fair peer-review system, which is all easy to use. Visit http://www.dovepress.com/testimonials.php to read real quotes from published authors.

Submit your manuscript here: http://www.dovepress.com/international-journal-of-chronic-obstructive-pulmonary-disease-journal 